\title{
Model Information
}

Data Set

Response Variable

Response Distribution

Link Function

Variance Function

Variance Matrix

Estimation Technique

Degrees of Freedom Method

\section{D.DADOS_AC_MTT}

$\mathrm{mtt}$

Gaussian

Identity

Default

Diagonal

Restricted Maximum Likelihood

Residual

\begin{tabular}{|l|r|l|}
\hline \multicolumn{3}{|c|}{ Class Level Information } \\
\hline Class & Levels & Values \\
\hline trat & 3 & CIV CIV + tt-farnesol Control \\
\hline bloco & 3 & 123 \\
\hline
\end{tabular}

Number of Observations Read

Number of Observations Used

\begin{tabular}{|c|c|c|}
\hline \multicolumn{3}{|c|}{ Dimensions } \\
\hline Covariance Parameters & & 1 \\
\hline Columns in $X$ & & 7 \\
\hline Columns in Z & & 0 \\
\hline Subjects (Blocks in V) & & 1 \\
\hline Max Obs per Subject & & 9 \\
\hline \multicolumn{3}{|c|}{ Optimization Information } \\
\hline Optimization Technique & None & \\
\hline Parameters & 6 & \\
\hline Lower Boundaries & 1 & \\
\hline Upper Boundaries & 0 & \\
\hline Fixed Effects & Not Profiled & \\
\hline
\end{tabular}

\begin{tabular}{|c|c|}
\hline \multicolumn{2}{|c|}{ Fit Statistics } \\
\hline -2 Res Log Likelihood & 28.41 \\
\hline AIC (smaller is better) & 40.41 \\
\hline AICC (smaller is better) & 124.41 \\
\hline BIC (smaller is better) & 36.73 \\
\hline
\end{tabular}




\begin{tabular}{|l|r|r|r|r|}
\hline \multicolumn{7}{|c|}{ Type III Tests of Fixed Effects } & Pr $>$ F \\
\hline Effect & Num DF & Den DF & F Value & 0.0414 \\
\hline bloco & 2 & 4 & 7.83 & 0.0013 \\
\hline trat & 2 & 4 & 52.81 & 0.81 \\
\hline
\end{tabular}

\begin{tabular}{|c|c|c|c|c|c|}
\hline \multicolumn{6}{|c|}{ trat Least Squares Means } \\
\hline Tratamento & Estimate & $\begin{array}{r}\text { Standard } \\
\text { Error }\end{array}$ & $D F$ & $t$ Value & $\operatorname{Pr}>|t|$ \\
\hline CIV & 60.6667 & 2.8122 & 4 & 21.57 & $<.0001$ \\
\hline $\mathrm{CIV}+\mathrm{tt}$-farnesol & 89.9500 & 2.8122 & 4 & 31.99 & $<.0001$ \\
\hline Control & 100.00 & 2.8122 & 4 & 35.56 & $<.0001$ \\
\hline
\end{tabular}

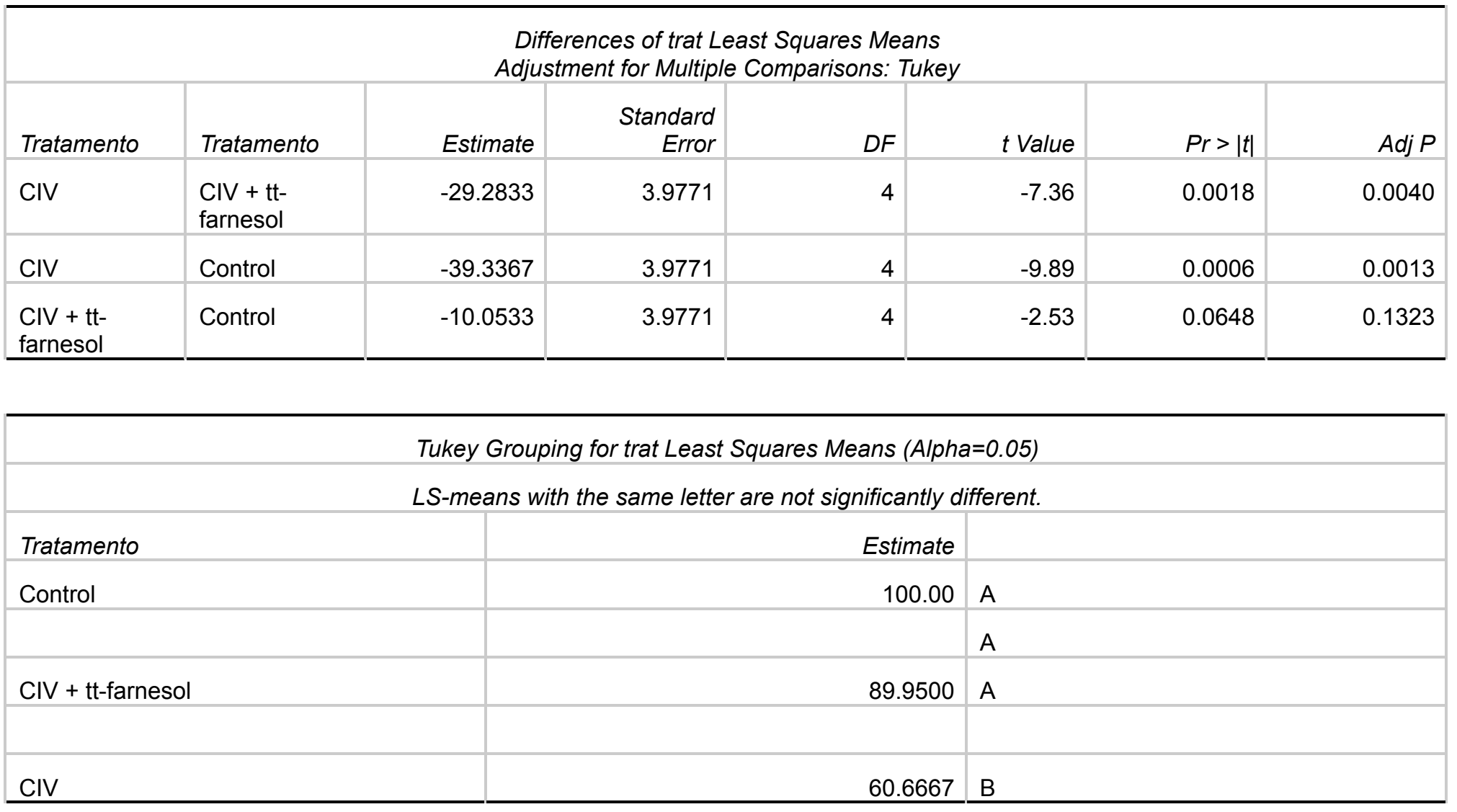


Analysis Variable : $m t t$ Toxicidade (MTT)

\begin{tabular}{|c|c|c|c|c|c|}
\hline Tratamento & NObs & Mean & Std Dev & $\begin{array}{l}\text { Upper } 95 \% \\
\text { CL for Mean }\end{array}$ & $\begin{array}{l}\text { Lower } 95 \% \\
\text { CL for Mean }\end{array}$ \\
\hline CIV & 3 & 60.67 & 10.43 & 86.59 & 34.75 \\
\hline $\mathrm{CIV}+\mathrm{tt}$-farnesol & 3 & 89.95 & 7.26 & 107.98 & 71.92 \\
\hline Control & 3 & 100.00 & 8.47 & 121.04 & 78.97 \\
\hline
\end{tabular}

\begin{tabular}{|r|r|r|r|}
\hline Obs & Tratamento & Bloco & Toxicidade (MTT) \\
\hline 1 & Control & 1 & 91.76 \\
\hline 2 & Control & 2 & 99.57 \\
\hline 3 & Control & 3 & 108.68 \\
\hline 4 & CIV + tt-farnesol & 1 & 81.78 \\
\hline 5 & CIV + tt-farnesol & 2 & 95.66 \\
\hline 6 & CIV + tt-farnesol & 3 & 92.41 \\
\hline 7 & CIV & 1 & 49.89 \\
\hline 8 & CIV & 2 & 70.72 \\
\hline 9 & CIV & 3 & 61.39 \\
\hline
\end{tabular}

